\title{
Ab initio Undersampled Phase Retrieval
}

\section{S. Marchesini}

\section{Advanced Light Source, Lawrence Berkeley National Lab. 1 Cyclotron rd. Berkeley CA, 94610}

Any object on earth has two fundamental properties: it is finite, and it is made of atoms. Structural information about an object can be obtained from diffraction amplitude measurements that account for either one of these traits. Nyquist-sampling of the Fourier amplitudes is sufficient to image single particles of finite size at any resolution. Atomic resolution data is routinely used to image molecules replicated in a crystal structure. Here we report an algorithm [1] that requires neither information, but uses the fact that an image of a natural object is compressible [2]. Intended applications include tomographic diffractive imaging, crystallography, powder diffraction, small angle x-ray scattering and random Fourier amplitude measurements.

\section{References}

\section{[1] S. Marchesini arXiv:0809.2006}

[2] M. L. Moravec, J. K. Romberg, R. G. Baraniuk, Wavelets XII. Proc. SPIE 6701, 670120 (2007).
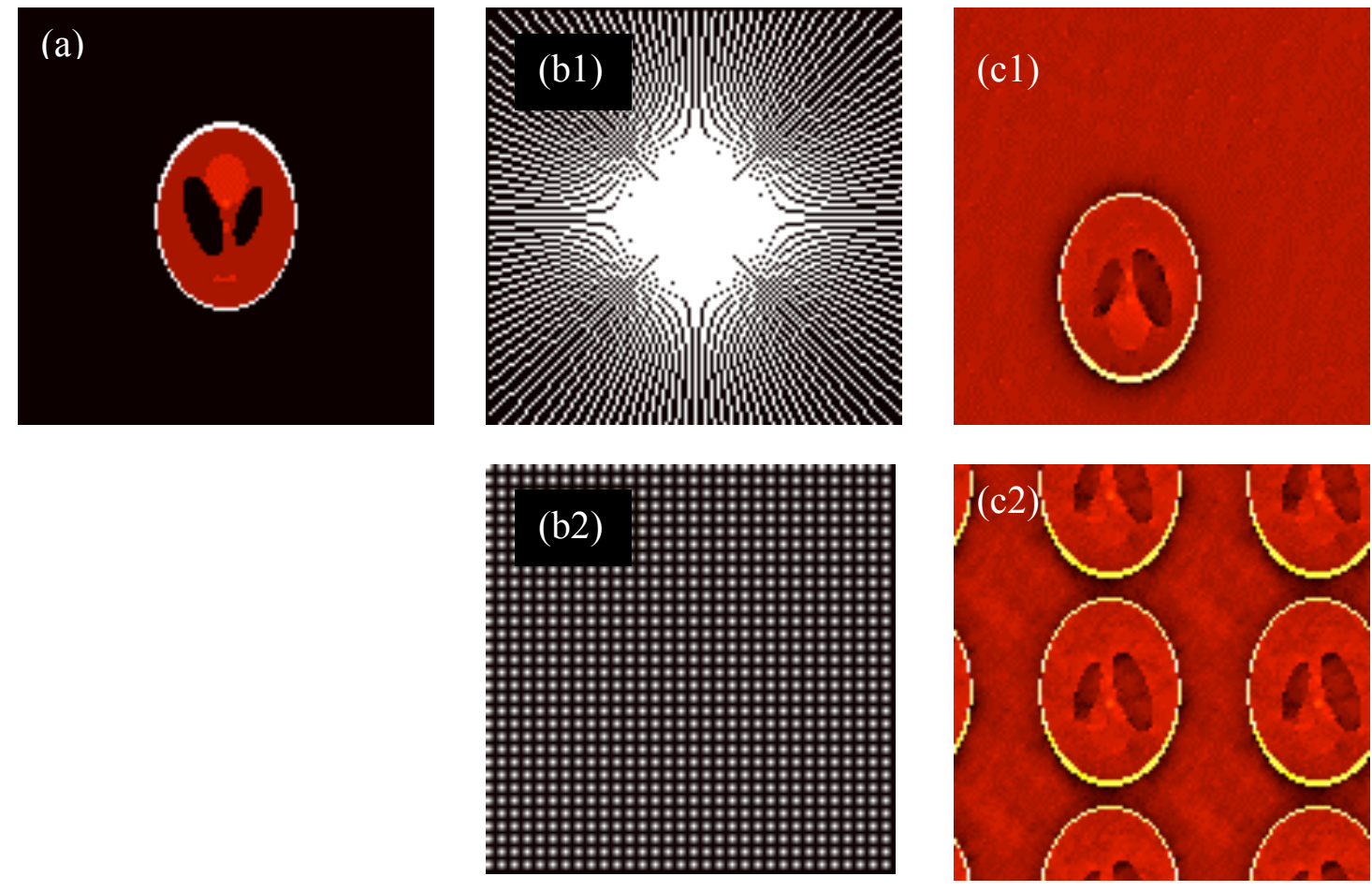

Fig.1 A two dimensional piecewise smooth test object (a) is reconstructed almost exactly (c) from incomplete Fourier amplitude measurements (b) by sparsifying the gradient of the object (minimizing the total variation) [1]. The dark points in (b) represent missing Fourier components, (b1) represents missing fourier measurements in a tomographic geometry, (b2) represents Fourier measurements at half the critical sampling rate. 